\title{
The Nicolas and Robin inequalities with sums of two squares
}

\author{
William D. Banks • Derrick N. Hart • \\ Pieter Moree • C. Wesley Nevans
}

Received: 12 October 2007 / Accepted: 26 February 2008 / Published online: 15 August 2008

(C) The Author(s) 2008. This article is published with open access at Springerlink.com

\begin{abstract}
In 1984, G. Robin proved that the Riemann hypothesis is true if and only if the Robin inequality $\sigma(n)<e^{\gamma} n \log \log n$ holds for every integer $n>5040$, where $\sigma(n)$ is the sum of divisors function, and $\gamma$ is the Euler-Mascheroni constant. We exhibit a broad class of subsets $\mathcal{S}$ of the natural numbers such that the Robin inequality holds for all but finitely many $n \in \mathcal{S}$. As a special case, we determine the finitely many numbers of the form $n=a^{2}+b^{2}$ that do not satisfy the Robin inequality. In fact, we prove our assertions with the Nicolas inequality $n / \varphi(n)<e^{\gamma} \log \log n$; since $\sigma(n) / n<n / \varphi(n)$ for $n>1$ our results for the Robin inequality follow at once.
\end{abstract}

Keywords Nicolas inequality $\cdot$ Robin inequality $\cdot$ Sums of two squares

Mathematics Subject Classification (2000) $\quad 11 \mathrm{Y} 35 \cdot 11 \mathrm{~A} 25$

Communicated by U. Zannier.

W. D. Banks · D. N. Hart · C. W. Nevans

Department of Mathematics, University of Missouri, Columbia, MO 65211, USA

e-mail: bbanks@math.missouri.edu

D. N. Hart

e-mail: hart@math.missouri.edu

C. W. Nevans

e-mail: nevans@math.missouri.edu

P. Moree $(\varangle)$

Max-Planck-Institut für Mathematik, Vivatsgasse 7, 53111 Bonn, Germany

e-mail: moree@mpim-bonn.mpg.de 


\section{Introduction}

Let $\varphi(n)$ denote the Euler function. In 1903 Landau (see [4, pp. 217-219]) showed that

$$
\varlimsup_{n \rightarrow \infty} \frac{n}{\varphi(n) \log \log n}=e^{\gamma},
$$

where $\gamma$ is the Euler-Mascheroni constant. Eighty years later, in a highly interesting work, Nicolas [5] proved that the inequality

$$
\frac{n}{\varphi(n)}>e^{\gamma} \log \log n
$$

holds for infinitely many natural numbers $n$. Moreover, if $N_{k}$ denotes the product of the first $k$ primes, he proved that

$$
\frac{N_{k}}{\varphi\left(N_{k}\right)}>e^{\gamma} \log \log N_{k}
$$

holds for every $k \geqslant 1$ on the Riemann hypothesis (RH). Assuming RH is false, he also showed there are both infinitely many $k$ for which this inequality holds and infinitely many $k$ for which it does not hold. To acknowledge the many contributions of Nicolas to this subject, we denote by $\mathcal{N}$ the set of numbers $n \in \mathbb{N}$ that satisfy the Nicolas inequality:

$$
\frac{n}{\varphi(n)}<e^{\gamma} \log \log n
$$

The principal aim of this paper is to exhibit a broad class of infinite subsets $\mathcal{S} \subset \mathbb{N}$ such that this inequality holds for all but finitely many $n \in \mathcal{S}$. This class includes a set that contains all natural numbers which can be expressed as a sum of two squares.

Let $\sigma(n)$ be the sum of divisors function. The analogue of (1) for this function was obtained by Gronwall [2], who proved that

$$
\varlimsup_{n \rightarrow \infty} \frac{\sigma(n)}{n \log \log n}=e^{\gamma}
$$

Robin [7] showed that if RH is true, then the Robin inequality:

$$
\frac{\sigma(n)}{n}<e^{\gamma} \log \log n
$$

holds for every integer $n>5040$, whereas if RH is false, then this inequality fails for infinitely many $n$. We denote by $\mathcal{R}$ the set of numbers $n \in \mathbb{N}$ that satisfy (3). In view of the elementary inequality

$$
\frac{\sigma(n)}{n}<\frac{n}{\varphi(n)} \quad(n>1),
$$


it is clear that $\mathcal{N} \subset \mathcal{R}$. Thus, for the class of subsets $\mathcal{S} \subset \mathbb{N}$ considered in the present paper, the Robin inequality holds for all but finitely many $n \in \mathcal{S}$.

Our work was originally inspired by a recent paper of Choie et al. [1], which establishes the inclusion in $\mathcal{R}$ of various infinite subsets of the natural numbers $\mathbb{N}$. In particular, in [1] it is shown that $\mathcal{R}$ contains every square-free number $n>30$, every odd integer $n>9$, every powerful number $n>36$, and every integer $n>1$ not divisible by the fifth power of some prime. As a consequence it follows that the RH holds iff the Robin inequality holds for all natural numbers $n$ divisible by the fifth power of some prime. Note that this criterion does not have the restriction $n \geqslant 5041$. Another "5041-free" criterion was given earlier by Lagarias [3], who showed that RH is true iff

$$
\sigma(n) \leqslant H_{n}+e^{H_{n}} \log H_{n}
$$

where

$$
H_{n}=\sum_{j \leqslant n} \frac{1}{j} \quad(n \geqslant 1)
$$

To state our results more precisely, let $\mathbb{P}$ denote the set of prime numbers, and for any subset $\mathcal{A} \subset \mathbb{P}$, put

$$
\pi_{\mathcal{A}}(x)=\#\{p \leqslant x: p \in \mathcal{A}\} .
$$

Let $\mathcal{P}$ be an arbitrary (fixed) subset of $\mathbb{P}$ such that

$$
\bar{\delta}=\varlimsup_{x \rightarrow \infty} \frac{\pi_{\mathcal{P}}(x)}{\pi(x)}<1 \text { and } \underline{\delta}=\varliminf_{x \rightarrow \infty} \frac{\pi_{\mathcal{P}}(x)}{\pi(x)}>0,
$$

where $\pi(x)=\#\{p \leqslant x\}$ as usual. Let $\mathcal{Q}$ denote the complementary set of primes (i.e., $\mathcal{Q}=\mathbb{P} \backslash \mathcal{P})$, and note that

$$
\varlimsup_{x \rightarrow \infty} \frac{\pi_{\mathcal{Q}}(x)}{\pi(x)}=1-\underline{\delta}<1 \text { and } \underline{\lim }_{x \rightarrow \infty} \frac{\pi_{\mathcal{Q}}(x)}{\pi(x)}=1-\bar{\delta}>0 .
$$

In this paper, we work with the set $\mathcal{S}=\mathcal{S}(\mathcal{P})$ defined by

$$
\mathcal{S}=\left\{n \in \mathbb{N}: \text { if } p \in \mathcal{Q} \text { and } p \mid n, \text { then } p^{2} \mid n\right\}
$$

Our main result is the following:

Theorem 1 The set $\mathcal{N}$ contains all but finitely many of the numbers in $\mathcal{S}$.

Corollary 1 Of the numbers $n$ which do not satisfy the Nicolas inequality, all but finitely many are divisible by a prime $q \in \mathcal{Q}$ such that $q^{2} \nmid n$. 
In particular, for any fixed $a, m \in \mathbb{N}$ with $\operatorname{gcd}(a, m)=1$, one can put

$$
\mathcal{P}=\{p \in \mathbb{P}: p \not \equiv a \quad(\bmod m)\}
$$

and apply Corollary 1 to deduce the following:

Corollary 2 Of the numbers $n$ which do not satisfy the Nicolas inequality, all but finitely many are divisible by a prime $q \equiv a(\bmod m)$ such that $q^{2} \nmid n$.

In Sect. 3 we examine more closely the special case

$$
\mathcal{P}=\{p \in \mathbb{P}: p \equiv 1 \quad(\bmod 4)\} \cup\{2\}
$$

Note that the corresponding set $\mathcal{S}$ contains all natural numbers of the form $n=$ $a^{2}+b^{2}$ (since, by a theorem of Fermat, every prime $q \equiv 3(\bmod 4)$ appears with even multiplicity in the prime factorization of $n$ if and only if $n$ can be written as a sum of two squares). Using effective bounds from [6] on the number of primes in arithmetic progressions modulo 4 , we are able to determine the set $\mathcal{S} \backslash \mathcal{N}$ completely, leading to:

Theorem 2 The set $\mathcal{S} \backslash \mathcal{N}$ contains precisely 347 natural numbers. In particular, there are precisely 246 numbers which can be expressed as a sum of two squares and such that the Nicolas inequality (2) does not hold, the largest of which is the number 52509581344222812810 .

As an application, we obtain the unconditional result that

$$
\{1,2,4,5,8,9,10,16,18,20,36,72,180,360,720\}
$$

is a complete list of those natural numbers which can be expressed as a sum of two squares and such that the Robin inequality (3) does not hold; this result is consistent with the truth of the Riemann Hypothesis.

Results like those of Theorem 2 can be established for certain quadratic forms other than $a^{2}+b^{2}$. For example, using similar techniques one finds that there are precisely 261 numbers that can be expressed in the form $n=a^{2}+3 b^{2}$ and for which the Nicolas inequality (2) does not hold, the largest of which is the number 397999936131188090700.

Throughout the paper, any implied constants in the symbols $O, \ll, \gg$ and $\asymp$ depend (at most) on the set $\mathcal{P}$ and are absolute otherwise. We recall that for positive functions $f, g$ the notations $f=O(g), f \ll g$ and $g \gg f$ are all equivalent to the assertion that $f \leqslant c g$ for some constant $c>0$, and the notation $f \asymp g$ means that $f \ll g$ and $g \ll f$. 


\section{Proof of Theorem 1}

For every natural number $n$ we put

$$
F(n)=\frac{n}{\varphi(n)}=\prod_{p \mid n} \frac{p}{p-1} .
$$

Note that

$$
F(n)=F(\kappa(n)) \text { and } \omega(n)=\omega(\kappa(n)),
$$

where $\omega(n)$ is the number of distinct prime divisors of $n$, and $\kappa(n)$ is the square-free kernel of $n$ :

$$
\kappa(n)=\prod_{p \mid n} p
$$

Let

$$
\mathcal{N}^{\circ}=\mathbb{N} \backslash \mathcal{N}=\left\{n \in \mathbb{N}: F(n) \geqslant e^{\gamma} \log \log n\right\},
$$

and for every integer $k \geqslant 0$, let

$$
\mathcal{V}_{k}=\{n \in \mathbb{N}: \omega(n) \geqslant k\} \text { and } \mathcal{W}_{k}=\mathcal{S} \cap \mathcal{N}^{\circ} \cap \mathcal{V}_{k}
$$

Since $\mathcal{V}_{0}=\mathbb{N}$, Theorem 1 is the assertion that $\mathcal{W}_{0}=\mathcal{S} \cap \mathcal{N}^{\circ}$ is a finite set. In view of the next lemma, it suffices to show that $\mathcal{W}_{k}=\varnothing$ for some $k$.

Lemma 1 For every $k \geqslant 0, \mathcal{W}_{0} \backslash \mathcal{W}_{k}$ is a finite set.

Since $\omega(n)<k$ and $F(n) \geqslant e^{\gamma} \log \log n$ for all $n \in \mathcal{W}_{0} \backslash \mathcal{W}_{k}$, Lemma 1 is an immediate consequence of the following:

Lemma 2 For every constant $K>0$, there are at most finitely many natural numbers $n$ such that $\omega(n) \leqslant K$ and $F(n) \geqslant e^{\gamma} \log \log n$.

Proof If $\bar{p}_{1}, \bar{p}_{2}, \ldots$ is the sequence of consecutive prime numbers, then for any such number $n$ we have

$$
\prod_{j \leqslant K} \frac{\bar{p}_{j}}{\bar{p}_{j}-1} \geqslant \prod_{p \mid n} \frac{p}{p-1}=F(n) \geqslant e^{\gamma} \log \log n
$$

this shows that $n$ is bounded by a constant which depends only on $K$. 
For every natural number $n$, let

$$
s(n)=\left(\prod_{\substack{p \mid n \\ p \in \mathcal{P}}} p\right)\left(\prod_{\substack{q \mid n \\ q \in \mathcal{Q}}} q^{2}\right),
$$

and put

$$
\mathcal{Y}=\{n \in \mathbb{N}: n=s(n)\} .
$$

Note that $\mathcal{Y} \subset \mathcal{S}$. The following statements are elementary:

$\left(\mathscr{C}_{1}\right)$ if $n=p m$ with $p \in \mathcal{P}$ and $p \nmid m$, then $n \in \mathcal{Y}$ if and only if $m \in \mathcal{Y}$;

$\left(\mathscr{C}_{2}\right)$ if $n=q^{2} m$ with $q \in \mathcal{Q}$ and $q \nmid m$, then $n \in \mathcal{Y}$ if and only if $m \in \mathcal{Y}$;

$\left(\mathscr{C}_{3}\right) \quad s(n) \in \mathcal{S}$ for all $n$;

$\left(\mathscr{C}_{4}\right) \quad \kappa(s(n))=\kappa(n)$ for all $n$;

$\left(\mathscr{C}_{5}\right) \quad s(n) \mid n$ for all $n \in \mathcal{S}$; in particular, $s(n) \leqslant n$.

Lemma 3 If $\mathcal{W}_{k} \neq \varnothing$ and $m_{k}$ is the least integer in $\mathcal{W}_{k}$, then $m_{k} \in \mathcal{Y}$.

Proof Clearly, $s\left(m_{k}\right) \in \mathcal{S}$ by $\left(\mathscr{C}_{3}\right)$. Combining $\left(\mathscr{C}_{4}\right)$ with $(7)$ one sees that

$$
F(s(n))=F(n) \text { and } \omega(s(n))=\omega(n) \quad(n \in \mathbb{N}) \text {. }
$$

Then, using $\left(\mathscr{C}_{5}\right)$ it follows that

$$
F\left(s\left(m_{k}\right)\right)=F\left(m_{k}\right) \geqslant e^{\gamma} \log \log m_{k} \geqslant e^{\gamma} \log \log s\left(m_{k}\right),
$$

which shows that $s\left(m_{k}\right) \in \mathcal{N}^{\circ}$. Finally, $s\left(m_{k}\right) \in \mathcal{V}_{k}$ since

$$
\omega\left(s\left(m_{k}\right)\right)=\omega\left(m_{k}\right) \geqslant k .
$$

Thus, we have shown that $s\left(m_{k}\right) \in \mathcal{S} \cap \mathcal{N}^{\circ} \cap \mathcal{V}_{k}=\mathcal{W}_{k}$. Since $m_{k}$ is the least integer in $\mathcal{W}_{k}$, the equality $m_{k}=s\left(m_{k}\right)$ follows from $\left(\mathscr{C}_{5}\right)$, hence $m_{k} \in \mathcal{Y}$.

Next, for every integer $k \geqslant 0$ let

$$
\mathcal{Z}_{k}=\{n \in \mathbb{N}: \Omega(n)=k\} \text { and } \mathcal{T}_{k}=\mathcal{N}^{\circ} \cap \mathcal{Y} \cap \mathcal{Z}_{k}
$$

Here, $\Omega(n)$ is the number of prime divisors of $n$, counted with multiplicity. Using Lemma 3 one sees that if $\mathcal{W}_{\ell} \neq \varnothing$ and $m_{\ell}$ is the least integer in $\mathcal{W}_{\ell}$, then $m_{\ell} \in \mathcal{T}_{k}$ for some $k \geqslant \ell$; in particular,

$$
\bigcup_{k \geqslant \ell} \mathcal{T}_{k}=\varnothing \Longrightarrow \mathcal{W}_{\ell}=\varnothing
$$


As we mentioned earlier, in order to prove Theorem 1 it suffices to show that $\mathcal{W}_{\ell}=\varnothing$ for some $\ell$, hence it is enough to show that $\mathcal{T}_{k} \neq \varnothing$ for at most finitely many integers $k \geqslant 0$.

When $\mathcal{T}_{k} \neq \varnothing$ we shall use the following notation. Let $n_{k}$ denote the least integer in $\mathcal{T}_{k}$. Let $\widehat{p}_{k}$ be the largest prime $p \in \mathcal{P}$ that divides $n_{k}$, and put $\widehat{p}_{k}=1$ if no such prime exists. Similarly, let $\widehat{q}_{k}$ be the largest prime $q \in \mathcal{Q}$ that divides $n_{k}$, and set $\widehat{q}_{k}=1$ if no such prime exists. Finally, let

$$
P_{k}^{+}=\max \left\{\widehat{p}_{k}, \widehat{q}_{k}\right\} \text { and } P_{k}^{-}=\min \left\{\widehat{p}_{k}, \widehat{q}_{k}\right\}
$$

Note that $P_{k}^{+}$is the largest prime factor of $n_{k}$.

Lemma 4 Suppose $\mathcal{T}_{k} \neq \varnothing$ :

(i) if $p \in \mathcal{P}$ with $p<\widehat{p}_{k}$, then $p \mid n_{k}$;

(ii) if $q \in \mathcal{Q}$ with $q<\widehat{q}_{k}$, then $q \mid n_{k}$.

Proof Suppose on the contrary that $p \in \mathcal{P}$ with $p<\widehat{p}_{k}$ and $p \nmid n_{k}$. Since $n_{k}=s\left(n_{k}\right)$ we can write $n_{k}=\widehat{p}_{k} m$ with $\widehat{p}_{k} \nmid m$. Put $n^{*}=p m$. Since $n_{k} \in \mathcal{N}^{\circ}, F(p)>F\left(\widehat{p}_{k}\right)$, and $n^{*}<n_{k}$, it follows that

$$
F\left(n^{*}\right)=F(p) F(m)>F\left(\widehat{p}_{k}\right) F(m)=F\left(n_{k}\right) \geqslant e^{\gamma} \log \log n_{k}>e^{\gamma} \log \log n^{*},
$$

where we have used the fact that $F$ is multiplicative; this shows that $n^{*} \in \mathcal{N}^{\circ}$. As $n_{k} \in \mathcal{Y},\left(\mathscr{C}_{1}\right)$ implies that $n^{*} \in \mathcal{Y}$. Finally, since $\Omega$ is (completely) additive, we see that

$$
\Omega\left(n^{*}\right)=\Omega(m)+1=\Omega\left(n_{k}\right)=k,
$$

which shows that $n^{*} \in \mathcal{Z}_{k}$, and thus $n^{*} \in \mathcal{N}^{\circ} \cap \mathcal{Y} \cap \mathcal{Z}_{k}=\mathcal{T}_{k}$. But this is impossible since $n^{*}<n_{k}$ (the least number in $\mathcal{T}_{k}$ ), and this contradiction completes our proof of (i). Using $\left(\mathscr{C}_{2}\right)$, the proof of (ii) is similar; we omit the details.

Lemma 5 Suppose that $\mathcal{T}_{k} \neq \varnothing$ and $\widehat{p}_{k}<\widehat{q}_{k}$. Then there is at most one prime $p \in \mathcal{P}$ such that $\widehat{p}_{k}<p<\widehat{q}_{k}$.

Proof Suppose on the contrary that there are two primes $p_{1}, p_{2} \in \mathcal{P}$ such that $\widehat{p}_{k}<$ $p_{1}<p_{2}<\widehat{q}_{k}$. Since $n_{k}=s\left(n_{k}\right)$ we can write $n_{k}=\widehat{q}_{k}^{2} m$, and it is clear that $\operatorname{gcd}(m$, $\left.p_{1} p_{2} \widehat{q}_{k}\right)=1$. Put $n^{*}=p_{1} p_{2} m$. Since $n_{k} \in \mathcal{N}^{\circ}, F\left(p_{1} p_{2}\right)>F\left(\widehat{q}_{k}^{2}\right)$, and $n^{*}<n_{k}$, we have

$F\left(n^{*}\right)=F\left(p_{1} p_{2}\right) F(m)>F\left(\widehat{q}_{k}^{2}\right) F(m)=F\left(n_{k}\right) \geqslant e^{\gamma} \log \log n_{k}>e^{\gamma} \log \log n^{*}$,

which shows that $n^{*} \in \mathcal{N}^{\circ}$. As $n_{k} \in \mathcal{Y},\left(\mathscr{C}_{1}\right)$ implies that $n^{*} \in \mathcal{Y}$. Finally, since

$$
\Omega\left(n^{*}\right)=\Omega(m)+2=\Omega\left(n_{k}\right)=k,
$$

we see that $n^{*} \in \mathcal{Z}_{k}$, and thus $n^{*} \in \mathcal{N}^{\circ} \cap \mathcal{Y} \cap \mathcal{Z}_{k}=\mathcal{T}_{k}$. But this is impossible since $n^{*}<n_{k}$, and this contradiction implies the result. 
Lemma 6 Suppose that $\mathcal{T}_{k} \neq \varnothing$ and $\widehat{p}_{k}>\widehat{q}_{k}$. Let $p$ be the largest prime in $\mathcal{P}$ that is less than $\widehat{p}_{k}$, and let $q$ be the smallest prime in $\mathcal{Q}$ that is greater than $\widehat{q}_{k}$. Then $q>p / 2$.

Proof Suppose on the contrary that $q \leqslant p / 2$. Since $n_{k}=s\left(n_{k}\right)$ and $p \mid n_{k}$ (by Lemma 4) but $q \nmid n_{k}$ (since $\left.q>\widehat{q}_{k}\right)$, we can write $n_{k}=p \widehat{p}_{k} m$, where $\operatorname{gcd}\left(m, p \widehat{p}_{k} q\right)=$ 1. Put $n^{*}=q^{2} m$. As in the proofs of Lemmas 4 and 5, we see that $n^{*} \in \mathcal{Y} \cap \mathcal{Z}_{k}$. Since $p<\widehat{p}_{k}$ and $q \leqslant p / 2$, we have

$$
F\left(p \widehat{p}_{k}\right)=\frac{p \widehat{p}_{k}}{(p-1)\left(\widehat{p}_{k}-1\right)}<\frac{p^{2}}{(p-1)^{2}}<\frac{q}{q-1}=F\left(q^{2}\right)
$$

therefore,

$$
F\left(n^{*}\right)=F\left(q^{2}\right) F(m)>F\left(p \widehat{p}_{k}\right) F(m)=F\left(n_{k}\right) \geqslant e^{\gamma} \log \log n_{k}>e^{\gamma} \log \log n^{*},
$$

which shows that $n^{*} \in \mathcal{N}^{\circ}$. Thus, $n^{*} \in \mathcal{N}^{\circ} \cap \mathcal{Y} \cap \mathcal{Z}_{k}=\mathcal{T}_{k}$. But this is impossible since $n^{*}<n_{k}$, and this contradiction implies the result.

As mentioned above, in order to prove Theorem 1 it suffices to show that $\mathcal{T}_{k} \neq \varnothing$ for at most finitely many integers $k \geqslant 0$. Arguing by contradiction, we shall assume that the set

$$
\mathcal{K}=\left\{k \geqslant 0: \mathcal{T}_{k} \neq \varnothing\right\}
$$

has infinitely many elements.

Since $\Omega\left(n_{k}\right)=k$, we see that $n_{k} \rightarrow \infty$ as $k \rightarrow \infty$ with $k \in \mathcal{K}$; using Lemma 2 it follows that $\omega\left(n_{k}\right) \rightarrow \infty$ as well, and therefore $P_{k}^{+} \rightarrow \infty$.

We claim that

$$
\widehat{p}_{k} \asymp \widehat{q}_{k} \quad(k \in \mathcal{K}),
$$

which by (8) is equivalent to

$$
P_{k}^{+} \asymp P_{k}^{-} \quad(k \in \mathcal{K}) .
$$

To see this, we express $\mathcal{K}$ as a disjoint union $\mathcal{A} \cup \mathcal{B}$, where $\mathcal{A}$ [resp. $\mathcal{B}$ ] is the set of numbers $k \in \mathcal{K}$ for which $\widehat{p}_{k}<\widehat{q}_{k}$ [resp. $\widehat{p}_{k}>\widehat{q}_{k}$ ]. To prove (9) it suffices to show:

$\left(\mathscr{D}_{1}\right) \quad \widehat{p}_{k} \gg \widehat{q}_{k}$ for all $k \in \mathcal{A}$;

$\left(\mathscr{D}_{2}\right) \quad \widehat{p}_{k} \ll \widehat{q}_{k}$ for all $k \in \mathcal{B}$.

We use the following result, which is an easy consequence of the prime number theorem:

Lemma 7 Let $c_{\mathcal{P}}=\bar{\delta} / \underline{\delta}$ and $c_{\mathcal{Q}}=(1-\underline{\delta}) /(1-\bar{\delta})$. For every $\varepsilon>0$ there is a number $x_{0}(\varepsilon)$ such that for all $x>x_{0}(\varepsilon)$ : 
(i) if $p$ is the smallest prime in $\mathcal{P}$ greater than $x$, then $p \leqslant\left(c_{\mathcal{P}}+\varepsilon\right) x$;

(ii) if $q$ is the smallest prime in $\mathcal{Q}$ greater than $x$, then $q \leqslant\left(c_{\mathcal{Q}}+\varepsilon\right) x$;

(iii) if $p$ is the largest prime in $\mathcal{P}$ less than $x$, then $p \geqslant\left(c_{\mathcal{P}}^{-1}-\varepsilon\right) x$;

(iv) if $q$ is the largest prime in $\mathcal{Q}$ less than $x$, then $q \geqslant\left(c_{\mathcal{Q}}^{-1}-\varepsilon\right) x$.

To prove $\left(\mathscr{D}_{1}\right)$ we can assume that $\mathcal{A}$ is an infinite set. Let $k \in \mathcal{A}$, so that $\widehat{p}_{k}<\widehat{q}_{k}$. Since $\widehat{q}_{k}=P_{k}^{+} \rightarrow \infty$ as $k \rightarrow \infty$ with $k \in \mathcal{A}$, the assertion $\left(\mathscr{D}_{1}\right)$ then follows from Lemmas 5 and 7.

To prove $\left(\mathscr{D}_{2}\right)$ we can assume that $\mathcal{B}$ is an infinite set. Let $k \in \mathcal{B}$, so that $\widehat{p}_{k}>\widehat{q}_{k}$. Let $p, q$ be defined as in Lemma 6. Since $\widehat{p}_{k}=P_{k}^{+} \rightarrow \infty$ as $k \rightarrow \infty$ with $k \in \mathcal{B}$, on combining Lemmas 6 and 7 it follows that

$$
\widehat{p}_{k} \ll p \ll q \ll \widehat{q}_{k},
$$

which proves $\left(\mathscr{D}_{2}\right)$ and completes our Proof of (9).

Next, for every $n \in \mathbb{N}$ let

$$
\omega_{\mathcal{P}}(n)=\#\{p \in \mathcal{P}: p \mid n\} \quad \text { and } \omega_{\mathcal{Q}}(n)=\#\{q \in \mathcal{Q}: q \mid n\}
$$

We claim that

$$
\omega_{\mathcal{P}}\left(n_{k}\right) \asymp \omega_{\mathcal{Q}}\left(n_{k}\right) \quad(k \in \mathcal{K}) .
$$

Indeed, by Lemma 4 it follows that $\omega_{\mathcal{P}}\left(n_{k}\right)=\pi_{\mathcal{P}}\left(\widehat{p}_{k}\right)$ and $\omega_{\mathcal{Q}}\left(n_{k}\right)=\pi_{\mathcal{Q}}\left(\widehat{q}_{k}\right)$. Therefore, using the prime number theorem together with (4), (5) and (9) we have

$$
\omega_{\mathcal{P}}\left(n_{k}\right)=\pi_{\mathcal{P}}\left(\widehat{p}_{k}\right) \asymp \frac{\widehat{p}_{k}}{\log \widehat{p}_{k}} \asymp \frac{\widehat{q}_{k}}{\log \widehat{q}_{k}} \asymp \pi_{\mathcal{Q}}\left(\widehat{q}_{k}\right)=\omega_{\mathcal{Q}}\left(n_{k}\right),
$$

which proves (11).

Finally, we need the following relation:

$$
\log \kappa\left(n_{k}\right) \asymp \omega\left(n_{k}\right) \log \omega\left(n_{k}\right) \quad(k \in \mathcal{K}) .
$$

To prove this, observe that Definition (8) and Lemma 4 together imply

$$
\prod_{p \leqslant P_{k}^{-}} p \mid \kappa\left(n_{k}\right) \text { and } \kappa\left(n_{k}\right) \mid \prod_{p \leqslant P_{k}^{+}} p
$$

Consequently,

$$
\sum_{p \leqslant P_{k}^{-}} \log p \leqslant \log \kappa\left(n_{k}\right) \leqslant \sum_{p \leqslant P_{k}^{+}} \log p
$$


and also

$$
\pi\left(P_{k}^{-}\right) \leqslant \omega\left(n_{k}\right) \leqslant \pi\left(P_{k}^{+}\right)
$$

By the prime number theorem, for either choice of the sign \pm we have

$$
\sum_{p \leqslant P_{k}^{ \pm}} \log p \sim P_{k}^{ \pm} \text {and } \pi\left(P_{k}^{ \pm}\right) \sim \frac{P_{k}^{ \pm}}{\log P_{k}^{ \pm}} \quad(k \rightarrow \infty, k \in \mathcal{K}),
$$

therefore in view of (10) we see that

$$
\log \kappa\left(n_{k}\right) \asymp P_{k}^{+} \quad \text { and } \quad \omega\left(n_{k}\right) \asymp \frac{P_{k}^{+}}{\log P_{k}^{+}},
$$

and (12) follows immediately.

Now we come to the heart of the argument. To complete the proof of Theorem 1, we seek a contradiction to our assumption that $\mathcal{K}$ is an infinite set. For this, it is enough to prove both of the following statements with a suitably chosen real number $\varepsilon>0$ :

$\left(\mathscr{E}_{1}\right)$ the inequality $n_{k} \leqslant \kappa\left(n_{k}\right)^{1+\varepsilon}$ holds for at most finitely many $k \in \mathcal{K}$;

$\left(\mathscr{E}_{2}\right)$ the inequality $n_{k}>\kappa\left(n_{k}\right)^{1+\varepsilon}$ holds for at most finitely many $k \in \mathcal{K}$.

In view of (11) and (12), there is a constant $C>1$ such that the inequalities

$$
\omega_{\mathcal{P}}\left(n_{k}\right) \leqslant(C-1) \omega_{\mathcal{Q}}\left(n_{k}\right)
$$

and

$$
\log \kappa\left(n_{k}\right) \leqslant C \omega\left(n_{k}\right) \log \omega\left(n_{k}\right)
$$

both hold if $k$ is sufficiently large. Let $C$ be fixed, and put $\varepsilon=C^{-3}$.

To prove $\left(\mathscr{E}_{1}\right)$, we suppose on the contrary that $n_{k} \leqslant \kappa\left(n_{k}\right)^{1+\varepsilon}$ holds for infinitely many $k \in \mathcal{K}$. Let $k$ be large, and put

$$
r=\omega_{\mathcal{P}}\left(n_{k}\right)=\pi_{\mathcal{P}}\left(\widehat{p}_{k}\right) \text { and } s=\omega_{\mathcal{Q}}\left(n_{k}\right)=\pi_{\mathcal{Q}}\left(\widehat{q}_{k}\right)
$$

By what we have already seen it is clear that $\min \{r, s\} \rightarrow \infty$ as $k \rightarrow \infty$ with $k \in \mathcal{K}$, thus by (13) we have

$$
r \leqslant(C-1) s
$$

if $k$ is large enough. By Lemma 4 and the fact that $n_{k} \in \mathcal{Y}$, it follows that

$$
n_{k}=\left(\prod_{\substack{p \leqslant \widehat{p}_{k} \\ p \in \mathcal{P}}} p\right)\left(\prod_{\substack{q \leqslant \widehat{q}_{k} \\ q \in \mathcal{Q}}} q^{2}\right) \text { and } \kappa\left(n_{k}\right)=\left(\prod_{\substack{p \leqslant \widehat{p}_{k} \\ p \in \mathcal{P}}} p\right)\left(\prod_{\substack{q \leqslant \widehat{q}_{k} \\ q \in \mathcal{Q}}} q\right) .
$$


Hence, our assumption that $n_{k} \leqslant \kappa\left(n_{k}\right)^{1+\varepsilon}$ implies that

$$
\kappa\left(n_{k}\right) \geqslant\left(\frac{n_{k}}{\kappa\left(n_{k}\right)}\right)^{1 / \varepsilon}=\left(\prod_{\substack{q \leqslant \widehat{q}_{k} \\ q \in \mathcal{Q}}} q\right)^{1 / \varepsilon} .
$$

If $\bar{p}_{1}, \bar{p}_{2}, \ldots$ is the sequence of consecutive prime numbers, then by the prime number theorem (and recalling our choice of $\varepsilon$ ) we derive that

$$
\log \kappa\left(n_{k}\right) \geqslant C^{3} \sum_{\substack{q \leqslant \widehat{q}_{k} \\ q \in \mathcal{Q}}} \log q \geqslant C^{3} \sum_{p \leqslant \bar{p}_{s}} \log p \sim C^{3} \bar{p}_{s} \sim C^{3} s \log s
$$

as $k \rightarrow \infty$ with $k \in \mathcal{K}$. On the other hand, using (14), (15) and the fact that $\omega\left(n_{k}\right)=$ $r+s$, it follows that

$$
\log \kappa\left(n_{k}\right) \leqslant C(r+s) \log (r+s) \leqslant C^{2} s \log (C s) \sim C^{2} s \log s .
$$

Since $C^{3}>C^{2}$, these two inequalities for $\log \kappa\left(n_{k}\right)$ lead to a contradiction once $k$ is sufficiently large, and this completes the proof of $\left(\mathscr{E}_{1}\right)$.

To prove $\left(\mathscr{E}_{2}\right)$ we use some ideas from Choie et al. [1]. Suppose that $n_{k}>\kappa\left(n_{k}\right)^{1+\varepsilon}$, and put $t=\omega\left(n_{k}\right)$. We claim that either

$$
\sum_{p \leqslant \bar{p}_{t}} \log p<(1+\varepsilon)^{-1 / 2} \bar{p}_{t}
$$

or

$$
\bar{p}_{t} \leqslant \exp (2 / \log (1+\varepsilon))
$$

Assuming the claim, it is easy to see that $\omega\left(n_{k}\right)$ is bounded above by a constant $K$ that depends only on $\varepsilon$. By Lemma $2, n_{k}$ can take only finitely many distinct values, which implies $\left(\mathscr{E}_{2}\right)$.

To prove the claim, assume that (17) fails:

$$
\log \left(\bar{p}_{1} \cdots \bar{p}_{t}\right)=\sum_{p \leqslant \bar{p}_{t}} \log p \geqslant(1+\varepsilon)^{-1 / 2} \bar{p}_{t}
$$

Thanks to Rosser and Schoenfeld [8] it is known that

$$
\prod_{p \leqslant x} \frac{p}{p-1} \leqslant e^{\gamma}\left(\log x+\frac{1}{\log x}\right) \quad(x>1)
$$


Therefore, taking $x=\bar{p}_{t}$ and noting that $\kappa\left(n_{k}\right) \geqslant \bar{p}_{1} \cdots \bar{p}_{t}$, we derive that

$$
\begin{aligned}
e^{\gamma}\left(\log \bar{p}_{t}+\frac{1}{\log \bar{p}_{t}}\right) & \geqslant \prod_{j=1}^{t} \frac{\bar{p}_{j}}{\bar{p}_{j}-1} \geqslant \frac{n_{k}}{\varphi\left(n_{k}\right)} \geqslant e^{\gamma} \log \log n_{k} \\
& >e^{\gamma} \log \left((1+\varepsilon) \log \kappa\left(n_{k}\right)\right) \\
& \geqslant e^{\gamma} \log \left((1+\varepsilon) \log \left(\bar{p}_{1} \cdots \bar{p}_{t}\right)\right) \\
& \geqslant e^{\gamma} \log \left((1+\varepsilon)^{1 / 2} \bar{p}_{t}\right)=e^{\gamma}\left(\log \bar{p}_{t}+0.5 \log (1+\varepsilon)\right) ;
\end{aligned}
$$

that is,

$$
\frac{1}{\log \bar{p}_{t}} \geqslant 0.5 \log (1+\varepsilon),
$$

which is equivalent to (18). This proves the claim and completes our proof of Theorem 1.

\section{Proof of Theorem 2}

We continue to use the notation of the previous section, but we focus on the special case that

$$
\begin{array}{ll}
\mathcal{P}=\{p \in \mathbb{P}: p \equiv 1 & (\bmod 4)\} \cup\{2\}, \\
\mathcal{Q}=\{q \in \mathbb{P}: q \equiv 3 \quad(\bmod 4)\}
\end{array}
$$

Note that the corresponding set $\mathcal{S}$ contains every natural number that can be expressed as a sum of two squares. As before, we write

$$
\mathcal{T}_{k}=\left\{n \in \mathbb{N}: F(n) \geqslant e^{\gamma} \log \log n, n=s(n), \text { and } \Omega(n)=k\right\}
$$

and put

$$
\mathcal{K}=\left\{k \geqslant 0: \mathcal{T}_{k} \neq \varnothing\right\} .
$$

Lemma 8 If $k \in \mathcal{K}$, then $P_{k}^{-}<50000$.

Proof For every real number $x \geqslant 10$, let

- $g_{\mathcal{P}}(x)=$ the smallest prime in $\mathcal{P}$ greater than $x$;

- $g_{\mathcal{Q}}(x)=$ the smallest prime in $\mathcal{Q}$ greater than $x$;

- $\ell_{\mathcal{P}}(x)=$ the largest prime in $\mathcal{P}$ less than $x$;

- $\ell_{\mathcal{Q}}(x)=$ the largest prime in $\mathcal{Q}$ less than $x$. 
Also, put

$$
\vartheta_{\mathcal{P}}(x)=\sum_{\substack{p \leqslant x \\ p \in \mathcal{P}}} \log p \text { and } \vartheta_{\mathcal{Q}}(x)=\sum_{\substack{q \leqslant x \\ q \in \mathcal{Q}}} \log q
$$

Using the explicit bounds of Theorems 1 and 2 of Ramaré and Rumely [6], we see that the inequalities

$$
0.49 x<\vartheta_{\mathcal{P}}(x)<0.51 x \text { and } 0.49 x<\vartheta_{\mathcal{Q}}(x)<0.51 x
$$

hold for all $x \geqslant 45000$ (note that $\vartheta_{\mathcal{P}}(x)=\log 2+\theta(x ; 4,1)$ and $\vartheta_{\mathcal{Q}}(x)=\theta(x ; 4,3)$ in the notation of [6]). Consequently, for any $x \geqslant 50000$ we have

$$
\frac{49}{51} x<\ell_{\mathcal{P}}(x)<x<g_{\mathcal{P}}(x)<\frac{51}{49} x
$$

and

$$
\frac{49}{51} x<\ell_{\mathcal{Q}}(x)<x<g_{\mathcal{Q}}(x)<\frac{51}{49} x
$$

Now suppose that $P_{k}^{-} \geqslant 50000$. Using Lemma 5 and the preceding bounds we have

$$
\widehat{q}_{k}<g_{\mathcal{P}}\left(g_{\mathcal{P}}\left(\widehat{p}_{k}\right)\right)<\left(\frac{51}{49}\right)^{2} \widehat{p}_{k}
$$

On the other hand, by Lemma 6 we have

$$
\frac{51}{49} \widehat{q}_{k}>g_{\mathcal{Q}}\left(\widehat{q}_{k}\right)>\frac{1}{2} \ell_{\mathcal{P}}\left(\widehat{p}_{k}\right)>\frac{49}{102} \widehat{p}_{k} .
$$

Hence, it follows that

$$
0.92 \widehat{q}_{k}<\widehat{p}_{k}<2.2 \widehat{q}_{k}
$$

By Lemma 4 it is clear that

$$
\log \kappa\left(n_{k}\right)=\sum_{\substack{p \leqslant \widehat{p}_{k} \\ p \in \mathcal{P}}} \log p+\sum_{\substack{q \leqslant \widehat{q}_{k} \\ q \in \mathcal{Q}}} \log q=\vartheta_{\mathcal{P}}\left(\widehat{p}_{k}\right)+\vartheta_{\mathcal{Q}}\left(\widehat{q}_{k}\right)
$$

On the other hand, arguing as in the proof of Theorem 1, it follows from (16) that

$$
\log \kappa\left(n_{k}\right) \geqslant \varepsilon^{-1} \vartheta_{\mathcal{Q}}\left(\widehat{q}_{k}\right)
$$

if $\varepsilon>0$ is fixed and $n_{k} \leqslant \kappa\left(n_{k}\right)^{1+\varepsilon}$. Combining the two preceding results with (19), we see that

$$
0.51\left(\widehat{p}_{k}+\widehat{q}_{k}\right) \geqslant \vartheta_{\mathcal{P}}\left(\widehat{p}_{k}\right)+\vartheta_{\mathcal{Q}}\left(\widehat{q}_{k}\right) \geqslant \varepsilon^{-1} \vartheta_{\mathcal{Q}}\left(\widehat{q}_{k}\right) \geqslant 0.49 \varepsilon^{-1} \widehat{q}_{k}
$$


since $P_{k}^{-} \geqslant 50000$; taking into account (20), we further have

$$
0.51(1+2.2) \widehat{q}_{k} \geqslant 0.51\left(\widehat{p}_{k}+\widehat{q}_{k}\right) \geqslant 0.49 \varepsilon^{-1} \widehat{q}_{k},
$$

which implies that $\varepsilon \geqslant 0.3002$. Thus, for the smaller value $\varepsilon=0.3$, we see that the condition $n_{k} \leqslant \kappa\left(n_{k}\right)^{1.3}$ implies $P_{k}^{-}<50000$.

On the other hand, if $n_{k}>\kappa\left(n_{k}\right)^{1.3}$, we put $t=\omega\left(n_{k}\right)$ as in the proof of Theorem 1 . Since $\varepsilon=0.3$, we derive from (17) and (18) that either

$$
\vartheta\left(\bar{p}_{t}\right)=\sum_{p \leqslant \bar{p}_{t}} \log p<(1.3)^{-1 / 2} \bar{p}_{t}<0.88 \bar{p}_{t}
$$

or

$$
\bar{p}_{t} \leqslant \exp (2 / \log 1.3)<2045 .
$$

Using again Theorems 1 and 2 of Ramaré and Rumely [6] (see also [8]), it is easy to see that the inequality (21) implies $\bar{p}_{t}<300$, hence the inequality $\bar{p}_{t}<2045$ holds in both cases. It follows that $t<310$, and therefore,

$$
\min \left\{\pi_{\mathcal{P}}\left(\widehat{p}_{k}\right), \pi_{\mathcal{Q}}\left(\widehat{q}_{k}\right)\right\}=\min \left\{\omega_{\mathcal{P}}\left(n_{k}\right), \omega_{\mathcal{Q}}\left(n_{k}\right)\right\} \leqslant \omega\left(n_{k}\right)=t<310,
$$

which implies that $P_{k}^{-}<5000$. This completes the proof.

Corollary 3 If $k \in \mathcal{K}$, then $k<10000$.

Proof For any $k \in \mathcal{K}$ we have

$$
k=\Omega\left(n_{k}\right)=\omega_{\mathcal{P}}\left(n_{k}\right)+2 \omega_{\mathcal{Q}}\left(n_{k}\right)=\pi_{\mathcal{P}}\left(\widehat{p}_{k}\right)+2 \pi_{\mathcal{Q}}\left(\widehat{q}_{k}\right) .
$$

If $P_{k}^{-}=\widehat{p}_{k}$ (i.e., $\widehat{p}_{k}<\widehat{q}_{k}$ ), then by Lemmas 5 and 8 it follows that

$$
\begin{aligned}
k & \leqslant \max _{p<50000}\left\{\pi_{\mathcal{P}}(p)+2 \pi_{\mathcal{Q}}\left(g_{\mathcal{P}}\left(g_{\mathcal{P}}(p)\right)\right)\right\} \\
& \leqslant \pi_{\mathcal{P}}(50000)+2 \pi_{\mathcal{Q}}\left(g_{\mathcal{P}}\left(g_{\mathcal{P}}(50000)\right)\right)=7718 .
\end{aligned}
$$

If $P_{k}^{-}=\widehat{q}_{k}$ (i.e., $\widehat{q}_{k}<\widehat{p}_{k}$ ), then by Lemmas 6 and 8 it follows that

$$
\begin{aligned}
k & \leqslant \max _{q<50000} \max _{\substack{p \in \mathbb{P} \\
\ell_{\mathcal{P}}(p)<2 g_{\mathcal{Q}}(q)}}\left\{\pi_{\mathcal{P}}(p)+2 \pi_{\mathcal{Q}}(q)\right\} \\
& =\max _{q<50000} \max _{\substack{p \in \mathbb{P} \\
\ell_{\mathcal{P}}(p)<2 g_{\mathcal{Q}}(q)}}\left\{1+\pi_{\mathcal{P}}\left(\ell_{\mathcal{P}}(p)\right)+2 \pi_{\mathcal{Q}}(q)\right\} \\
& \leqslant \max _{q<50000}\left\{1+\pi_{\mathcal{P}}\left(2 g_{\mathcal{Q}}(q)\right)+2 \pi_{\mathcal{Q}}(q)\right\} \\
& \leqslant 1+\pi_{\mathcal{P}}\left(2 g_{\mathcal{Q}}(50000)\right)+2 \pi_{\mathcal{Q}}(50000)=9951 .
\end{aligned}
$$

The result follows. 
Now let $\overline{\bar{p}}_{1}, \overline{\bar{p}}_{2}, \ldots$ be the sequence of consecutive primes in $\mathcal{P}$, and let $\overline{\bar{q}}_{1}, \overline{\bar{q}}_{2}, \ldots$ be the consecutive primes in $\mathcal{Q}$. For any integers $r, s \geqslant 0$, let

$$
N_{r, s}=\left(\prod_{i=1}^{r} \overline{\bar{p}}_{i}\right)\left(\prod_{j=1}^{s} \overline{\bar{q}}_{j}^{2}\right) .
$$

It is easy to see that $N_{r, s} \in \mathcal{Y}$ for all $r, s \geqslant 0$, and for every $k \in \mathcal{K}$ one has

$$
n_{k}=N_{r, s}, \quad \widehat{p}_{k}=\overline{\bar{p}}_{r}, \quad \widehat{q}_{k}=\overline{\bar{q}}_{s} \text { and } k=r+2 s,
$$

where $r=\omega_{\mathcal{P}}\left(n_{k}\right)$ and $s=\omega_{\mathcal{Q}}\left(n_{k}\right)$. By a straightforward computation, one verifies the following:

Lemma 9 If $r, s \geqslant 0$, then $N_{r, s} \in \mathcal{N}^{\circ}$ if and only if the pair $(r, s)$ lies in the set

$$
\begin{aligned}
\mathcal{X}= & \{(0,0),(1,0),(0,1),(2,0),(1,1),(2,1),(1,2),(3,1),(2,2),(4,1), \\
& (3,2),(2,3),(4,2),(3,3),(5,2),(4,3),(3,4),(5,3),(4,4),(6,3), \\
& (5,4),(4,5),(7,3),(6,4),(5,5),(7,4),(6,5),(7,5),(8,5)\} .
\end{aligned}
$$

We remark that, in view of Corollary 3, it suffices to check the condition $N_{r, s} \in \mathcal{N}^{\circ}$ only for those pairs $(r, s)$ with $r+2 s<10000$.

Corollary 4 If $k \in \mathcal{K}$, then $k \leqslant 18$.

Corollary 5 If $n \in \mathcal{S} \cap \mathcal{N}^{\circ}, r=\omega_{\mathcal{P}}(n)$ and $s=\omega_{\mathcal{Q}}(n)$, then $(r, s) \in \mathcal{X}$. In particular, $\omega(n) \leqslant 13$.

Proof Since

$$
F\left(N_{r, s}\right)=\left(\prod_{i=1}^{r} \frac{\overline{\bar{p}}_{i}}{\overline{\bar{p}}_{i}-1}\right)\left(\prod_{j=1}^{s} \frac{\overline{\bar{q}}_{j}}{\overline{\bar{q}}_{j}-1}\right) \geqslant\left(\prod_{\substack{p \mid n \\ p \in \mathcal{P}}} \frac{p}{p-1}\right)\left(\prod_{\substack{q \mid n \\ q \in \mathcal{Q}}} \frac{q}{q-1}\right)=F(n)
$$

and

$$
n \geqslant s(n)=\left(\prod_{\substack{p \mid n \\ p \in \mathcal{P}}} p\right)\left(\prod_{\substack{q \mid n \\ q \in \mathcal{Q}}} q^{2}\right) \geqslant\left(\prod_{i=1}^{r} \overline{\bar{p}}_{i}\right)\left(\prod_{j=1}^{s} \overline{\bar{q}} q_{j}^{2}\right)=N_{r, s}
$$

we have

$$
F\left(N_{r, s}\right) \geqslant F(n) \geqslant e^{\gamma} \log \log n \geqslant e^{\gamma} \log \log N_{r, s},
$$

which shows that $N_{r, s} \in \mathcal{N}^{\circ}$. 
We now turn to a description of our method for generating the elements of $\mathcal{S} \backslash \mathcal{N}=$ $\mathcal{S} \cap \mathcal{N}^{\circ}$. For any given $n \in \mathcal{S} \cap \mathcal{N}^{\circ}$ with $r=\omega_{\mathcal{P}}(n)$ and $s=\omega_{\mathcal{Q}}(n)$, we can write

$$
s(n)=p_{1} \cdots p_{r} q_{1}^{2} \cdots q_{s}^{2}
$$

where $p_{1}<\cdots<p_{r}$ are primes in $\mathcal{P}$ and $q_{1}<\cdots<q_{s}$ are primes in $\mathcal{Q}$. For fixed $i=1, \ldots, r$, let $\gamma_{i}$ be the largest non-negative integer such that the number

$$
\left(\prod_{\ell=1}^{i-1} \overline{\bar{p}}_{\ell}\right)\left(\prod_{\ell=i}^{r} \overline{\bar{p}}_{\ell+\gamma_{i}}\right)\left(\prod_{j=1}^{s} \overline{\bar{q}}_{j}^{2}\right)
$$

lies in $\mathcal{N}^{\circ}$, which exist by Lemma 2 . Using an argument similar to that in the proof of Lemma 4, one can deduce that

$$
\overline{\bar{p}}_{i} \leqslant p_{i} \leqslant \overline{\bar{p}}_{i+\gamma_{i}} \quad(i=1, \ldots, r)
$$

Similarly, for fixed $j=1, \ldots, s$, let $\delta_{j}$ be the largest non-negative integer such that the number

$$
\left(\prod_{i=1}^{r} \overline{\bar{p}}_{i}\right)\left(\prod_{\ell=1}^{j-1} \overline{\bar{q}}_{\ell}^{2}\right)\left(\prod_{\ell=j}^{s} \overline{\bar{q}}_{\ell+\delta_{j}}^{2}\right)
$$

lies in $\mathcal{N}^{\circ}$. Then,

$$
\overline{\bar{q}}_{j} \leqslant q_{j} \leqslant \overline{\bar{q}}_{j+\gamma_{j}}(j=1, \ldots, s) .
$$

Therefore, for fixed $(r, s) \in \mathcal{X}$, if $n \in \mathcal{S} \cap \mathcal{N}^{\circ}$ with $r=\omega_{\mathcal{P}}(n)$ and $s=\omega_{\mathcal{Q}}(n)$, then the number $s(n)$ must lie in the finite set $\mathcal{A}_{r, s}$ of integers of the form

$$
m=p_{1} \cdots p_{r} q_{1}^{2} \cdots q_{s}^{2}
$$

where $p_{1}<\cdots<p_{r}$ are primes in $\mathcal{P}, q_{1}<\cdots<q_{s}$ are primes in $\mathcal{Q}$, the primes $p_{i}$ and $q_{j}$ satisfy the bounds (22) and (23), and $m \in \mathcal{N}^{\circ}$. The set $\mathcal{A}_{r, s}$ can be explicitly determined by a numerical computation, and we obtain a finite list of "admissible" values for the quantity $s(n)$.

To determine explicitly all of the numbers $n \in \mathcal{S} \cap \mathcal{N}^{\circ}$ with $r=\omega_{\mathcal{P}}(n)$ and $s=\omega_{\mathcal{Q}}(n)$, for every $m \in \mathcal{A}_{r, s}$ we need to find all such numbers for which $s(n)=m$. To do this, factor $m$ as in (24). For fixed $i=1, \ldots, r$, let $\alpha_{i}$ be the largest integer such that the number $m p_{i}^{\alpha_{i}-1}$ lies in $\mathcal{N}^{\circ}$. Similarly, for fixed $j=1, \ldots, s$, let $\beta_{j}$ be the largest integer such that the number $m q_{j}^{\beta_{j}-1}$ lies in $\mathcal{N}^{\circ}$. Put

$$
M=m \cdot p_{1}^{\alpha_{1}-1} \cdots p_{r}^{\alpha_{r}-1} q_{1}^{\beta_{1}-1} \cdots q_{s}^{\beta_{s}-1}
$$


Then, it is easy to see that $m \mid n$ and $n \mid M$ for any $n \in \mathcal{S} \cap \mathcal{N}^{\circ}$ such that $s(n)=m$. Hence, $n$ can take only finitely many values which can be determined explicitly for each $m \in \mathcal{A}_{r, s}$.

For example, taking $r=s=2$ we find that

\{4410, 8820, 10890, 13230, 17640, 21780, 22050, 26460, 30870, 35280, 39690,

$44100,52920,61740,66150,70560,79380,88200,92610,105840,110250\}$

is a complete list of the numbers $n \in \mathcal{S} \backslash \mathcal{N}$ with $\omega_{\mathcal{P}}(n)=\omega_{\mathcal{Q}}(n)=2$. Examining the lists generated as $(r, s)$ varies over the pairs in $\mathcal{X}$, we are lead to the statement of Theorem 2.

\section{Evaluation of $\varlimsup_{n \in \mathcal{S}} \frac{n}{\varphi(n) \log \log n}$ and $\varlimsup_{n \in \mathcal{S}} \frac{\sigma(n)}{n \log \log n}$}

We conclude the paper by giving two propositions and two corollaries that yield the analogue of the work of Landau [4] and Gronwall [2] for any set $\mathcal{S}$ of the form (6) and for the set of natural numbers equal to a sum of two squares. In fact, Corollary 6 shows that Theorem 1 is nontrivial in the sense that $F(n) / \log \log n$ cannot be bounded away from $e^{\gamma}$ by any positive constant for all large $n \in \mathcal{S}$. We will use the notation $f(n)=o(g(n))$ to mean that $\lim _{n \rightarrow \infty} f(n) / g(n)=0$.

Proposition 1 Let $\left\{a_{n}\right\}$ be an infinite sequence of positive integers such that if we write $a_{n}=\prod_{p} p^{v(p, n)}$ we have:

(i) $\quad \kappa\left(a_{n}\right)=\prod_{p \leqslant n} p \quad$ (i.e., $\left.v(p, n)=0 \Longleftrightarrow p>n\right)$;

(ii) $a_{n}=\exp \left(n^{1+o(1)}\right)$;

(iii) $\lim _{n \rightarrow \infty} v(p, n)=\infty$ for each $p$.

Then,

$$
\lim _{n \rightarrow \infty} \frac{\sigma\left(a_{n}\right)}{a_{n} \log \log a_{n}}=e^{\gamma}
$$

Proof For all $n \geqslant 1$, let

$$
b_{n}=\prod_{p \leqslant n} p \quad \text { and } \quad c_{n}=\frac{\sigma\left(a_{n}\right)}{a_{n}} \frac{\varphi\left(b_{n}\right)}{b_{n}}
$$

and observe that (i) implies

$$
c_{n}=\left(\prod_{p \leqslant n} \frac{p^{v(p, n)+1}-1}{p^{v(p, n)}(p-1)}\right)\left(\prod_{p \leqslant n} \frac{p-1}{p}\right)=\prod_{p \leqslant n}\left(1-\frac{1}{p^{v(p, n)+1}}\right) .
$$


Since $v(p, n)+1 \geqslant 2$ for every prime $p \leqslant n$, we have for any $m \leqslant n$ :

$$
1 \geqslant c_{n}>\prod_{p \leqslant m}\left(1-\frac{1}{p^{v(p, n)+1}}\right) \prod_{p>m}\left(1-\frac{1}{p^{2}}\right) .
$$

Using (iii) we have for every fixed integer $m$ :

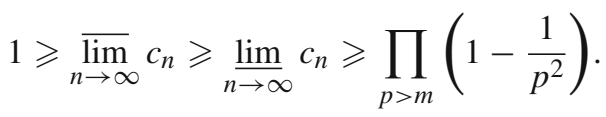

The product on the right tends to one as $m \rightarrow \infty$, hence $\lim _{n \rightarrow \infty} c_{n}=1$; therefore,

$$
\lim _{n \rightarrow \infty} \frac{\sigma\left(a_{n}\right)}{a_{n} \log n}=\lim _{n \rightarrow \infty} \frac{b_{n}}{\varphi\left(b_{n}\right) \log n} .
$$

Our assumption (ii) implies that $\log \log a_{n}=(1+o(1)) \log n$, and using Mertens' theorem (see, e.g., [8]) we have

$$
\frac{\varphi\left(b_{n}\right)}{b_{n}}=\prod_{p \leqslant n}\left(1-\frac{1}{p}\right)=(1+o(1)) \frac{e^{-\gamma}}{\log n}
$$

and the result follows.

Using similar ideas (and an easier argument) one can obtain the following analogue of Proposition 1 for the Euler totient function:

Proposition 2 Let $\left\{a_{n}\right\}$ be an infinite sequence of positive integers such that:

(i) $\kappa\left(a_{n}\right)=\prod_{p \leqslant n} p$;

(ii) $a_{n}=\exp \left(n^{1+o(1)}\right)$.

Then,

$$
\lim _{n \rightarrow \infty} \frac{a_{n}}{\varphi\left(a_{n}\right) \log \log a_{n}}=e^{\gamma}
$$

Corollary 6 For any set $\mathcal{S}$ defined by (6), we have

$$
\varlimsup_{n \in \mathcal{S}} \frac{\sigma(n)}{n \log \log n}=\varlimsup_{n \in \mathcal{S}} \frac{n}{\varphi(n) \log \log n}=e^{\gamma} .
$$

Proof Since

$$
\varlimsup_{n \rightarrow \infty} \frac{\sigma(n)}{n \log \log n}=\varlimsup_{n \rightarrow \infty} \frac{n}{\varphi(n) \log \log n}=e^{\gamma}
$$


by [2] and [4], respectively, it suffices to show that there is a sequence $\left\{a_{n}\right\}$ in $\mathcal{S}$ such that

$$
\lim _{n \rightarrow \infty} \frac{\sigma\left(a_{n}\right)}{a_{n} \log \log a_{n}}=\lim _{n \rightarrow \infty} \frac{a_{n}}{\varphi\left(a_{n}\right) \log \log a_{n}}=e^{\gamma} .
$$

Let $a_{1}=1$, and for every integer $n \geqslant 2$, let

$$
b_{n}=\prod_{p \leqslant n} p, \quad d_{n}=\left\lfloor n^{(\log n)^{-1 / 2}}\right\rfloor \quad \text { and } \quad a_{n}=b_{n}^{d_{n}} .
$$

It is easy to see that $d_{n} \geqslant 2$ for $n \geqslant 2, d_{n}=n^{o(1)}$, and $d_{n}$ tends to infinity with $n$. Clearly, $a_{n} \in \mathcal{S}$ for all $n \geqslant 1$, and by the Prime Number Theorem in the form $\sum_{p \leqslant x} \log p=x(1+o(1))$ as $x \rightarrow \infty$ we see that

$$
\log a_{n}=d_{n} \log b_{n}=n^{o(1)} \sum_{p \leqslant n} \log p=n^{1+o(1)} \quad(n \rightarrow \infty)
$$

The sequence $\left\{a_{n}\right\}$ therefore satisfies the hypotheses of Propositions 1 and 2, and the result follows.

Corollary 7 We have

$$
\varlimsup_{n=a^{2}+b^{2}} \frac{\sigma(n)}{n \log \log n}=\varlimsup_{n=a^{2}+b^{2}} \frac{n}{\varphi(n) \log \log n}=e^{\gamma} .
$$

Proof Defining $a_{n}$ for all $n \geqslant 1$ as in the proof of Corollary 6, it is easy to see that the sequence $\left\{a_{n}^{2}\right\}$ satisfies the hypotheses of Propositions 1 and 2; it follows that

$$
\varlimsup_{n=a^{2}} \frac{\sigma(n)}{n \log \log n}=\varlimsup_{n=a^{2}} \frac{n}{\varphi(n) \log \log n}=e^{\gamma},
$$

and this implies the stated result.

Open Access This article is distributed under the terms of the Creative Commons Attribution Noncommercial License which permits any noncommercial use, distribution, and reproduction in any medium, provided the original author(s) and source are credited.

\section{References}

1. Choie, Y.-J., Lichiardopol, N., Moree, P., Solé, P.: On Robin's criterion for the Riemann hypothesis. J. Théor Nombres Bord. 19, 351-366 (2007)

2. Gronwall, T.H.: Some asymptotic expressions in the theory of numbers. Trans. Am. Math. Soc. 14, 113122 (1913)

3. Lagarias, J.C.: An elementary problem equivalent to the Riemann hypothesis. Am. Math. Mon. 109, 534-543 (2002)

4. Landau, E.: Handbuch der Lehre von der Verteilung der Primzahlen. Teubner, Leipzig (1909) 
5. Nicolas, J.L.: Petites valeurs de la fonction d'Euler. J. Number Theory 17, 375-388 (1983)

6. Ramaré, O., Rumely, R.: Primes in arithmetic progressions. Math. Comp. 65, 397-425 (1996)

7. Robin, G.: Grandes valeurs de la fonction somme des diviseurs et hypothèse de Riemann. J. Math. Pures Appl. (9) 63, 187-213 (1984)

8. Rosser, J.B., Schoenfeld, L.: Approximate formulas for some functions of prime numbers Illinois. J. Math. 6, 64-94 (1962) 